\title{
A Mini-Thoracotomy Approach for Walking Veno-Arterial Extracorporeal Membranous Oxygenation
}

\author{
Eric Jeng ${ }^{1}$, Alex Parker ${ }^{2}$, and Mark Bleiweis ${ }^{3}$ \\ ${ }^{1}$ University of Florida \\ ${ }^{2}$ University of Florida Health \\ ${ }^{3}$ University of Florida COM
}

June 5, 2020

\begin{abstract}
Fulminant myocarditis is a rapidly progressive myocardial inflammation that commonly requires advanced therapies circulatory support. We report our management for a case of fulminant myocarditis and cardiogenic shock. The patient is a 36 year old gentleman who was admitted after a one week history of malaise. Upon admission he was lethargic with jugular venous distension to $10 \mathrm{~cm}$. He was taken immediately for a heart catheterization and intra-aortic balloon pump placement. There was no obstructive coronary disease, and hemodynamics were consistent with biventricular failure. After multidisciplinary evaluation, we elected to proceed with emergent extracorporeal membranous oxygenation (ECMO). We utilize a Protek Duo Rapid Deployment (LivaNova, Mirandola, Italy) which is inserted via modified Seldinger technique through the left ventricular apex, terminating in the ascending aorta. Percutaneous right IJ bicaval via a y-ed Avalon Elite (Getinge, Goteborg, Sweden) approach is employed for venous drainage (Figure 1). We believe that with this alternative ECMO cannulation platform, we address the multitude of drawbacks that plague peripherally cannulated extracorporeal circulatory support, minimizing patient deconditioning and upper/lower extremity over/under perfusion complications, while providing sternal sparring antegrade arterial flow with ventricular unloading/venting. For two weeks the patient was ambulatory, but because we were unable to obtain an adequate offer during this interval, we transitioned to a bridge to bridge therapy. This case highlights an alternate strategy for central walking VA ECMO in the rare presentation of one patient's progression from IABP to VA ECMO to durable BiVAD to heart transplantation during a single admission.
\end{abstract}

\section{TITLE PAGE}

I. Full title: A Mini-Thoracotomy Approach for Walking Veno-Arterial Extracorporeal Membranous Oxygenation

II. Running head: Walking Veno-Arterial Extracorporeal Membranous Oxygenation

III: Key words: perfusion; transplant

IV: Authors:

1. Eric I. Jeng, MD, MBA, University of Florida, College of Medicine, Division of Thoracic and Cardiovascular Surgery, Gainesville, FL

2. Alex M. Parker, MD, University of Florida, College of Medicine, Division of Cardiology, Gainesville, FL

3. Mark S. Bleiweis, MD, University of Florida, College of Medicine, Division of Thoracic and

Cardiovascular Surgery, Gainesville, FL 
V: No research scholarship awarded

VI: Manuscript is not currently under consideration elsewhere.

VII: Conflicts of Interest and Sources of Funding: All of the authors including Eric I. Jeng, Alex R. Parker, and Mark S. Bleiweis are in agreement with the content in the manuscript. There are no sources of financial support in the form of grants, equipment, and/or pharmaceutical items for this research. There are no potential conflicts of interest.

VIII: Word count for abstract is 248 . Word count for text body is 934 .

IX:

corresponding author:

Eric I. Jeng, MD, MBA

University of Florida

Division of Thoracic and Cardiovascular Surgery

PO Box 100287

Gainesville, FL 32610

ejeng2@gmail.com,

Phone: (310) 922-4265, (352) 265-0916, (352) 265-3292 (Fax)

\section{ABSTRACT}

Fulminant myocarditis is a rapidly progressive myocardial inflammation that commonly requires advanced therapies circulatory support. We report our management for a case of fulminant myocarditis and cardiogenic shock. The patient is a 36 year old gentleman who was admitted after a one week history of malaise. Upon admission he was lethargic with jugular venous distension to $>10 \mathrm{~cm}$. He was taken immediately for a heart catheterization which showed no obstructive coronary disease, and hemodynamics consistent with biventricular failure. After multidisciplinary evaluation, we elected to proceed with emergent extracorporeal membranous oxygenation (ECMO). We utilized a mini-thoracotomy approach for this cannulation. A Protek Duo Rapid Deployment (LivaNova, Mirandola, Italy) was inserted via modified Seldinger technique through the left ventricular apex, terminating in the ascending aorta. Percutaneous right IJ bicaval via a y-ed Avalon Elite (Getinge, Goteborg, Sweden) was employed for venous drainage (Figure 1). We believe that with this alternative ECMO cannulation platform, we can minimizing patient deconditioning and upper/lower extremity over/under perfusion complications, while providing sternal sparring antegrade arterial flow with ventricular unloading/venting. For two weeks the patient was ambulatory, but because we were unable to obtain an adequate offer during this interval, we transitioned to a bridge to bridge therapy with durable biventricular assist devices (VAD). The patient subsequently received an adequate donor offer one week after bi-VAD implantation, and underwent heart transplantation during the same admission. This case highlights an alternate strategy for central walking VA ECMO in a patient presenting with fulminant myocarditis and cardiogenic shock.

A Mini-Thoracotomy Approach for Walking Veno-Arterial Extracorporeal Membranous Oxygenation

Introduction

Fulminant myocarditis is a rapidly progressive inflammation of the myocardium (1). It requires aggressive and comprehensive management that commonly includes myocardial biopsy, inotropes, and advanced therapies for circulatory support (2). Herein, we report our surgical management strategy for a case of fulminant myocarditis and cardiogenic shock.

Case Report 
The patient is a 36 year old gentleman who was admitted to an outside institution after a one week history of malaise, and intermittent palpitations. He had no significant past medical or past surgical history. His initial work-up was significant for a troponin of 13 , and an LVEF of $20 \%$ on transthoracic echocardiogram (TTE). An immediate left heart catheterization was obtained which showed non-obstructive coronary disease. He was transferred to our center for advanced heart failure therapies. Upon admission he was lethargic, had jugular venous distension to $>10 \mathrm{~cm}$, and bilateral lower extremity edema. Repeat laboratory results were significant for a troponin of $>27 \mathrm{~K}, \mathrm{AST} / \mathrm{ALT} 687 / 506$, total bilirubin 1.2, INR 1.6, creatinine 1.65, Hgb/Hct 13.4/39.4. Bedside TTE showed biventricular dysfunction and an LVEF of 10\%. He was taken immediately for a right heart catheterization and intra-aortic balloon pump (IABP) placement. The hemodynamics with the IABP in place were a central venous pressure of $20 \mathrm{mmhg}$, pulmonary artery pressure of 46/32 $\mathrm{mmhg}$, and pulmonary capillary wedge of $32 \mathrm{mmhg}$. After heart team multidisciplinary evaluation, we elected to proceed with immediate application of extracorporeal membranous oxygenation (ECMO).

As in most centers, we customize our ECMO cannulation strategy based on the specific needs of the patient. We have employed various platforms including percutaneous femoral Veno-Arterial (VA) ECMO with distal perfusion, axillary arterial access ECMO, and direct central access ECMO. In those patients whereby, the etiology is of the cardiogenic shock is potentially recoverable, and a myocardial biopsy is necessary, we have utilized a central cannulation approach via a mini left anterior thoracotomy. A Protek Duo Rapid Deployment (LivaNova, Mirandola, Italy) is inserted via modified Seldinger technique through the left ventricular apex, terminating in the ascending aorta. This single cannula has proximal ports that drain the left ventricle, and a distal port that acts as central arterial cannula. With a direct left ventricular apex approach, we are aligned to simultaneously obtain a myocardial biopsy which can direct both treatment and prognosis. Percutaneous right IJ bicaval via a y-ed Avalon Elite (Getinge, Goteborg, Sweden) approach is employed for venous drainage. Our cannulation technique is diagrammed by Figure 1. Pathology from myocardial biopsy was consistent with lymphocytic myocarditis. Despite steroid treatment, and complete myocardial unloading, there was no recovery of right or left ventricular function. Medical review board discussion led to Status 1 listing. For two weeks the patient was ambulatory, and doing well, but had not yet received an adequate allograft offer. Beginning on VA ECMO day 15, the patient started requiring 1-2 unit of prbc transfusions daily, and so we modified our strategy to transition the patient to a durable ventricular assist platform. The patient underwent implantation of two Heartware (Medtronic, Minneapolis, MN) ventricular assist devices (VAD) for biventricular support. He tolerated the procedure well, was extubated postoperative day (POD) 1, and was ambulating by POD 3. On POD 8 we received and accepted an offer for a donor heart. The Bi-VADs and recipient heart were explanted and donor heart was implanted. Pathology of recipient heart was significant for inflammatory infiltrates and considerable myocyte necrosis. The patient was extubated and ambulating POD 0 from heart transplantation. He was discharged POD 16 after heart transplantation. The patient is now one year post transplantation and continues to do well.

\section{Discussion}

Patients with fulminant myocarditis require varying platforms of hemodynamic support (1). This group can be differentiated from the more benign uncomplicated myocarditis presentation as they have significantly lower systolic blood pressure, higher creatine kinase, wider QRS duration, lower left ventricular ejection fraction, thicker left ventricular posterior wall diameter, higher incidence of ST depression, and more ventricular tachycardia/ventricular fibrillation (1). Although several immunomodulatory or immunosuppressive therapies are prescribed in real-world practice for myocarditis, their effectiveness has not been clearly demonstrated. Patients with giant cell and eosinophilic histological subtypes of acute myocarditis and/or systemic autoimmune disorders and sarcoidosis seem to benefit most from immunosuppression (2). On the other hand, no clear evidence supports the use of immunosuppressive agents in patients with lymphocytic acute myocarditis (2). This finding was consistent with our patient (lymphocytic myocarditis) as he had no response to steroid therapy.

Mechanical circulatory support is often required to stabilize patients with acute fulminant myocarditis with cardiogenic shock (3). To that end, the balance between systemic circulatory support and cardiopulmonary 
unloading can have implications for short and long-term mortality. An over-distended left ventricular exposed to high myocardial stress, strain, work and oxygen consumption, as well as reduced coronary blood flow, will likely be unable to recover (4). Furthermore, while pulmonary edema may due to systemic inflammation mediated by shock and blood contact to artificial extracorporeal surfaces, high left ventricular filling pressures may also contribute (4). In this context, acute lung injury has been shown to significantly impact on prognosis in patients receiving VA ECMO, even after successful bridge-to-bridge therapy (5). We believe that with this alternative anterior left thoracotomy VA ECMO cannulation strategy, patient deconditioning is minimized, and we provide sternal sparring antegrade arterial flow with ventricular unloading/venting without the upper or lower extremity complications associated with axillary or femoral cannulation. This case highlights an alternative cannulation strategy for central walking VA ECMO in the rare presentation of fulminant myocarditis and cardiogenic shock, and one patient's progression from IABP to VA ECMO to durable BiVAD to heart transplantation (Figure 2) during a single admission.

\section{REFERENCES}

1. Wang Z, Wang Y, Lin H, Wang S, Cai X, Gao D. Early characteristics of fulminant myocarditis vs non-fulminant myocarditis: A meta-analysis.Medicine (Baltimore) . 2019;98(8):e14697. doi:10.1097/MD.0000000000014697

2. Ammirati E, Veronese G, Cipriani M, et al. Acute and Fulminant Myocarditis: a Pragmatic Clinical Approach to Diagnosis and Treatment.Curr Cardiol Rep . 2018;20(11):114. Published 2018 Sep 26. doi:10.1007/s11886-018-1054-z

3. Tschöpe C, Van Linthout S, Klein O, et al. Mechanical Unloading by Fulminant Myocarditis: LV-IMPELLA, ECMELLA, BI-PELLA, and PROPELLA Concepts. $J$ Cardiovasc Transl Res . 2019;12(2):116-123. doi:10.1007/s12265-018-9820-2

4. Donker DW, Brodie D, Henriques JPS, Broomé M. Left ventricular unloading during veno-arterial ECMO: a review of percutaneous and surgical unloading interventions. Perfusion . 2019;34(2):98-105. doi:10.1177/0267659118794112

5. Boulate D, Luyt CE, Pozzi M, et al. Acute lung injury after mechanical circulatory support implantation in patients on extracorporeal life support: an unrecognized problem. Eur J Cardiothorac Surg . 2013;44(3):544-550. doi:10.1093/ejcts/ezt125 


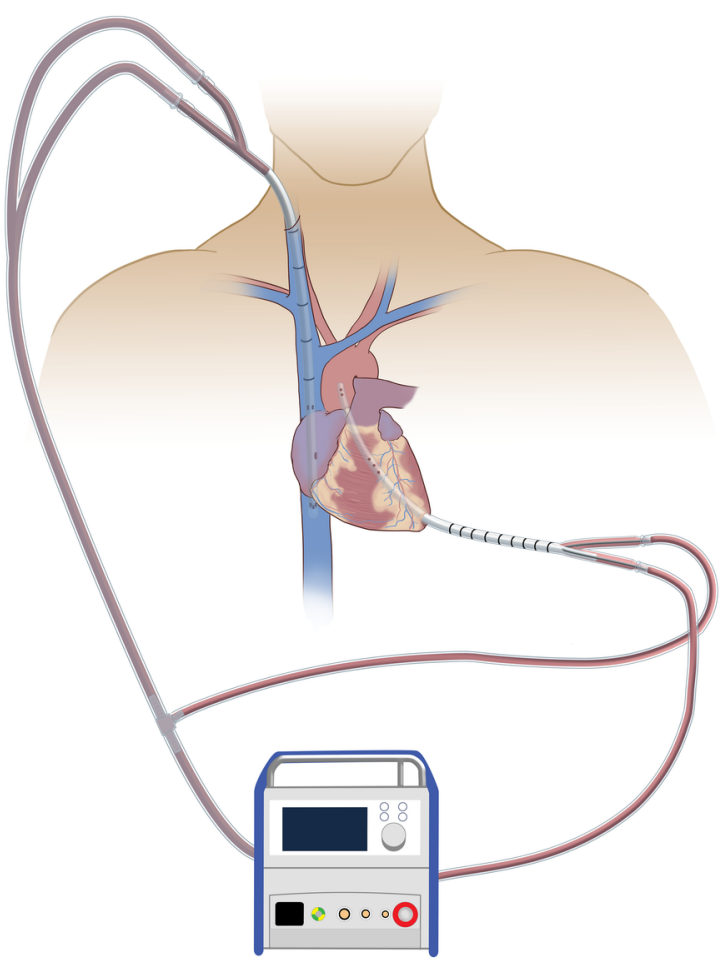

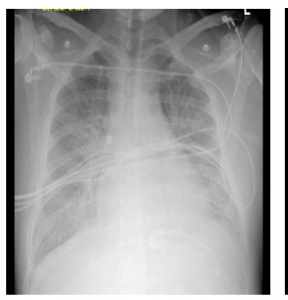

Preoperative

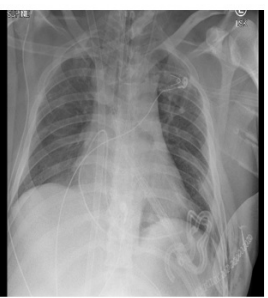

Protek Duo RD VA ECMO

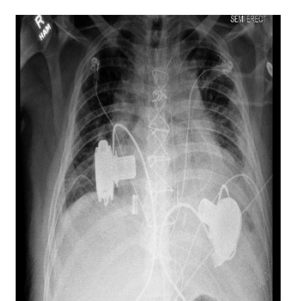

BiVAD Heartware

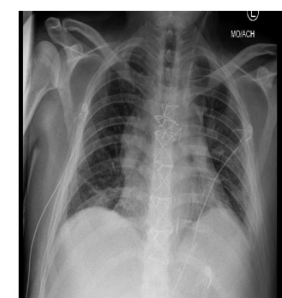

OHTx 